\title{
EchoGéo
}

$16 \mid 2011$

Échos de Turquie

\section{Géographie et media : des coopérations à} construire

Introduction

Alexis Sierra

\section{OpenEdition}

Journals

\section{Édition électronique}

URL : https://journals.openedition.org/echogeo/12439

DOI : 10.4000/echogeo.12439

ISSN : 1963-1197

\section{Éditeur}

Pôle de recherche pour l'organisation et la diffusion de l'information géographique (CNRS UMR 8586)

\section{Référence électronique}

Alexis Sierra, «Géographie et media : des coopérations à construire », EchoGéo [En ligne], 16 | 2011, mis en ligne le 04 juillet 2011, consulté le 25 août 2021. URL : http://journals.openedition.org/ echogeo/12439; DOI : https://doi.org/10.4000/echogeo.12439

Ce document a été généré automatiquement le 25 août 2021.

EchoGéo est mis à disposition selon les termes de la licence Creative Commons Attribution - Pas d'Utilisation Commerciale - Pas de Modification 4.0 International (CC BY-NC-ND) 


\title{
Géographie et media : des coopérations à construire
}

\author{
Introduction
}

Alexis Sierra

1 L'un des paradoxes de la géographie est d'être à la fois populaire et méconnue. Source d'exotisme, évoquant le voyage et l'aventure, partant le plus souvent du visuel, la géographie est en toile de fond de toute une série de programmes télévisuels depuis le bulletin météorologique jusqu'aux émissions d'évasion type C'est dans l'air ou Ushuaia. Cependant, contrairement à l'histoire par exemple, elle ne fait pas l'objet en soi de tels programmes. Le rapprochement de la figure quasi mythifiée du géographe de terrain avec celle du journaliste reporter est pourtant monnaie courante. Un café géographique ne s'interrogeait-il pas il y a quelques années sur le fait de savoir si Tintin, le héros de BD, reporter de profession, n'était pas géographe ${ }^{1}$ ? Et de constater que si le goût pour le voyage et pour la confrontation avec l'Autre était commun aux deux figures, Tintin utilisait peu les outils du géographe et analysait peu l'espace. Tintin est moins un géographe qu'il n'est l'objet d'une géographie à déconstruire. Entre un Elisée Reclus voyageur qui travaillait pour les guides touristiques Joanne de la maison Hachette et un Albert Londres, ou mutatis mutandis, entre un chercheur géographe et un grand reporter aujourd'hui, il y a en commun, non seulement la mobilité et la curiosité mais aussi les techniques d'interview, l'utilisation de la photographie ${ }^{2}$, l'utilisation de plans et de données pour se repérer et établir un contexte. Autrement dit, le travail de terrain semble proche. Il l'est d'autant plus, que les deux semblent traverser les champs de la connaissance, le journaliste comme le géographe puisant dans différents savoirs pour mener son investigation. Si lors de la restitution d'information, la description ultérieure de ce terrain peut encore être similaire c'est bien dans l'analyse exposée des faits que les deux figures s'écartent du fait des objectifs et de la formation.

2 La formation du journaliste aujourd'hui ne prête que peu d'attention à ces passerelles. Les écoles de journalisme dispensent une formation prioritairement orientée sur la communication. Celles de Lille (ESJ) ou de Paris (IPJ, CFJ), ne dispensent pas de cours de 
géographie bien qu'elles offrent des séminaires thématiques dans d'autres domaines comme l'économie ${ }^{3}$. Elles partent souvent du principe que le savoir académique est acquis avant l'école, ce qui peut être problématique quand la géographie disparait progressivement des premières années de Sciences Po par exemple. Certes, les épreuves du concours d'entrée introduisent une évaluation des connaissances en géographie mais le plus souvent présentées dans un ensemble appelé connaissance de l'actualité ou culture générale dans laquelle sont inclus l'histoire contemporaine, des éléments d'économie voire de droit. Le "savoir penser l'espace", notamment le travail d'articulation des échelles et des différentes spatialités qui sert au géographe pour comprendre une situation, ne semble pas fondamental.

Pourtant, les media ont multiplié l'utilisation des cartes de localisation. Pour éclairer une actualité, ils doivent faire appel à des spécialistes de certaines régions parmi lesquels des géographes. On se souvient ainsi que Bernard Hourcade avait été particulièrement sollicité lors du fort mouvement de contestation iranienne en 2009. $\mathrm{Au}$ demeurant, il a plus souvent été présenté par son titre (directeur de recherche au CNRS) que comme géographe. Plusieurs journaux développent un service infographique avec une composante cartographique. Ici également, les cartes servent au premier chef à localiser. Certains media utilisent parfois des systèmes d'information géographique. En 1992, après le passage de l'ouragan Andrew, le Miami Herald fut le premier journal à utiliser un SIG afin de comprendre le phénomène et montrer que le zonage établi en Floride augmentait le risque. Cette utilisation de l'outil géographique lui valut le prix Pulitzer en $1993^{4}$.

\footnotetext{
La géographie au service d'un média : l'expérience de géomarketing du Trome au Pérou

Le Trome est un petit journal populaire de Lima au Pérou proche de la presse à sensation. Au départ, la rédaction souhaite avoir à disposition un système de localisation à partir de la voirie pour aider les journalistes dans leur investigation : savoir se rendre sur les lieux d'un événement, situer cet événement. Cette première expérience met le journal en relation avec Vladimir Garcia, géographe de l'Université Catholique où il dispense un cours de cybercartographie. En 2005-2006, le journal s'interroge sur ses ventes et notamment sur l'absence de ventes dans certains districts. Le géographe et le journal décident de mettre en place une base de données géoréférencées du lectorat. Pour cela, le journal organise une loterie : pour participer les lecteurs doivent remplir un bordereau avec leur adresse. Grâce au système de localisation par rue, le géographe a pu identifier les différentes densités de lecteurs et en particulier les espaces vides. Dès lors, le Trome a fait une campagne de communication ciblée sur ces espaces. C'est ainsi que la rédaction a utilisé un outil géographique initialement destiné pour les journalistes en un outil pour développer ses ventes.
}

4 Alors que les liens entre géographie et journalisme semblent évidents, les coopérations ne semblent pas très développées. On peut donc s'interroger sur la légitimité de la géographie pour les medias, discipline à la fois nécessaire pour décrypter l'actualité et méconnue. Les difficultés à cerner ce qu'est la géographie, sans cesse fois posées, expliquent sans aucun doute sa faible présence dans les media. Cependant, il faudrait également s'interroger sur l'action des géographes eux-mêmes: investissent-ils les media et le décryptage de l'actualité ? Certains ont joué la carte de la médiatisation comme Yves Lacoste qui, en réhabilitant la géopolitique a donné à la géographie une certaine audience. D'autres lui ont emboîté le pas et nous en profitons pour rappeler que la revue EchoGéo a eu le souci dès l'origine d'investir l'actualité avec sa rubrique Sur 
le Vif. Dans un registre davantage pédagogique, des géographes ont pu construire des émissions pour l'enseignement à distance, en s'appuyant notamment sur la visualisation de cartes, comme Rodolph de Koninck ${ }^{5}$ l'avait fait au Québec.

Dans une autre voie, des géographes de formation ont pu se tourner vers le journalisme comme le montrent certains $\mathrm{CV}$ en ligne et la presse scientifique. Une recherche resterait à établir dans ce domaine. Le cas de Frank Tétart ${ }^{6}$, tout en ayant un parcours original, illustre parfaitement l'articulation entre les deux domaines. Initialement germaniste, il a étroitement mis en relation journalisme, géopolitique et cartographie, originellement en participant à l'émission d'Arte, Le Dessous des cartes et, depuis 2009, en participant à l'aventure des magazines Carto et Moyen-Orient. Le premier a pour but d'analyser les grandes questions d'actualité à travers l'analyse de cartes. Celles-ci sont de types différents, des cartes statistiques à des cartes historiques en passant par des cartes thématiques à toutes les échelles, elles permettent le plus souvent une analyse de situation, pour reprendre une terminologie vidalienne, avec une claire orientation géopolitique. La revue Moyen-Orient, comme l'indique son sous-titre, offre des études géopolitiques, géoéconomiques, géostratégiques des territoires et des sociétés du monde arabo-musulman.

6 L'expérience de Frank Tétart montre ainsi que la géographie peut devenir le support de commentaires journalistiques diffusés avec succès. Elle montre aussi les contraintes du journalisme: les techniques de diffusion et surtout le temps de préparation. Le journalisme implique une forte réactivité et la mobilisation de connaissances en un temps très bref pour répondre à un événement soudain. Le temps de la diffusion dans l'audiovisuel est également bref et ne supporte pas des messages trop complexes. La cartographie doit être simplifiée non seulement pour qu'une population profane à la géographie puisse se l'approprier mais également parce que, comme nous l'indique Frank Tétart, les techniques de diffusion télévisuelle limitent les possibilités. Ce temps du journalisme empêche enfin un retour d'expérience sur l'utilisation de la géographie par les journalistes eux-mêmes. Certains reporters que nous avons pu contacter ont spontanément estimé qu'ils n'avaient que peu à dire alors que dans les faits ils utilisent bien des savoirs géographiques. L'expérience du Dessous des Cartes montre cependant que la production de produits dérivés, notamment de dvd, peut favoriser une démarche réflexive.

7 Ainsi, à travers l'interview qui suit, se pose à la fois la question des finalités et de la vulgarisation de la géographie. Le lancement des magazines comme Carto et MoyentOrient, l'appétit grandissant pour les questions géopolitiques et environnementales en particulier, la diversification des media qui notamment par la Toile multiplient les possibilités de donner à voir les territoires que construit l'humanité donnent aux géographes les opportunités de faire reconnaître leurs compétences. 


\section{NOTES}

1. Tintin, une hergéographie, Cafés géographiques, 23 octobre 2001, présenté par JeanDominique Merchet (journaliste à Libération) et Jean-Louis Tissier (Professeur de géographie, Université de Paris XII), compte-rendu de Maud Lasseur

2. Voire de la vidéographie comme peut le montrer Béatrice Collignon.

3. D'après les programmes mis en ligne sur leur site web.

4. Mapping the News, David Herzog, Case Studies in GIS \& Journalism, ESRI Press, 2003, California.

5. Le monde à la carte, diffusé au Canada sur les ondes de Canal Savoir et de Télé-Québec

6. Tétart F., 2001. Le Dessous des cartes, originalité d'une émission géopolitique. Mémoire de DEA, sous la direction de Béatrice Giblin, Centre d'Analyse et de Recherche en géopolitique, Université de Paris VIII.

\section{AUTEUR}

\section{ALEXIS SIERRA}

Alexis Sierra est maître de conférences en géographie à l'université Cergy-Pontoise-IUFM.

alexisierra2001@yahoo.fr 